\title{
A Versatile all-Optical Parity-Time Signal Processing Device using a Bragg Grating Induced using Positive and Negative Kerr- Nonlinearity
}

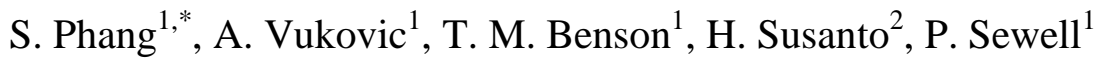 \\ ${ }^{1}$ The George Green Institute for Electromagnetics Research, Faculty of \\ Engineering, University of Nottingham, University Park, Nottingham NG7 2RD, \\ $U K$ \\ ${ }^{2}$ Department of Mathematical Sciences, University of Essex, Colchester CO4 3SQ, \\ $U K$
}

(*Corresponding author: E-mail: sendy.phang@nottingham.ac.uk)

\begin{abstract}
The properties of gratings with Kerr nonlinearity and PT symmetry are investigated in this paper. The impact of the gain and loss saturation on the response of the grating is analysed for different input intensities and gain/loss parameters. Potential applications of these gratings as switches, logic gates and amplifiers are also shown.
\end{abstract}

Keywords: Kerr-nonlinearity, gratings, balanced gain and loss

\section{Introduction}

Devices with balanced gain and loss can, under certain conditions, operate in a stable regime and can perform functionalities such as cloaking (Zhu et al. 2013), unidirectional invisibility (Lin et al. 2011; Regensburger et al. 2012), and power oscillation (Makris et al. 2008). These structures, also known as Parity-Time (PT) structures, require that the profile of complex refractive index $\hat{n}$ satisfy the condition $\hat{n}(-z)=\hat{n}^{*}(z)$, where $*$ denotes a complex conjugate operator. In other words, the PT-symmetric condition requires that the real and imaginary parts (gain/loss) of the refractive index are modulated as symmetric and anti-symmetric functions of space respectively (Chong et al. 2011; Lin et al. 2011; Rüter et al. 2010). The stable operating conditions are determined by the amount of gain and loss in the grating, whereby if the magnitudes of the gain and loss are equal, and 
exceed a certain critical amount, the grating operation becomes unstable (Chong et al. 2011; Nixon et al. 2012). This critical amount of gain/loss is commonly referred to as the Lasing and Coherent Perfect Absorber (CPAL) point (Chong et al. 2011; Phang et al. 2013b; Schindler et al. 2012), and operation above this point yields an exponential energy growth. In a linear PT grating structure, the CPAL occurs when the trajectories of a pole and a zero of the scattering matrix $\overline{\bar{S}}$ coincide on the real frequency axis in the complex frequency plane (Chong et al. 2011). It is also noted here, that several experimental works have been performed for different PT structures, i.e. coupled waveguide (Rüter et al. 2010), Bragg grating (Feng et al. 2013), lattices (Regensburger et al. 2012) and whisperinggallery mode structures (Feng et al. 2014; Hodaei et al. 2014; Peng et al. 2014) and demonstrated the predicted theoretical PT behaviour with low power signal and pump sources.

In this paper we focus on the one dimensional PT Bragg gratings. Reported PT Bragg gratings to date commonly have a constant phase grating combined with alternating layers of gain and loss (Kulishov et al. 2005; Lin et al. 2011; Phang et al. 2013b). The impact of Kerr-nonlinearity has also been analysed in the frequency domain in (Achilleos et al. 2012; Lin et al. 2011; Musslimani et al. 2008; Ramezani et al. 2010) assuming a non-saturable and frequency independent gain/loss material. In our recent paper we have analysed nonlinear PT Bragg gratings in time domain and have demonstrated that gain/loss saturation preserve the hysteresis properties of nonlinear PT Bragg grating, and that in the absence of gain/loss saturation hysteresis vanished (Phang et al. 2014). It is important to note that PT Bragg gratings (PTBG) have different diffraction properties depending on the side of excitation in that the transmittance is the same regardless of the side of excitations but reflectances are different. This is in contrast to the conventional Bragg grating (no gain/loss) which is a reciprocal structure. A consequence of this non-reciprocal operation of the PTBG is the unidirectional invisibility which occurs when the gain/loss parameter equals the modulation depth of the real part of the refractive index (Lin et al. 2011; Phang et al. 2013b).

In this paper we consider an extension of the grating investigated in (Brzozowski and Sargent 2000) in that we include PT symmetry condition. The structure is a nonlinear PT Bragg grating where the phase grating is intensity modulated by alternating layers of positive and negative Kerr-nonlinearity and 
combined with alternating layers of gain and loss. This means that for very low intensities the modulation of the real part of the refractive index is negligible and the grating is formed of purely gain and loss layers. As the intensity is increased, the phase grating is formed. This structure differs from one in (Phang et al. 2014) where the phase grating is constant and only the positive Kerr nonlinearity is introduced throughout the grating.

Nonlinear PT devices require two input beams, namely a strong pump beam to activate the Kerr nonlinearity and the probe beam. It is important to note that a passive grating structure, i.e. no gain and loss, with alternate sign of Kerr nonlinearity has been reported (Brzozowski and Sargent 2000) which offered alloptical limiting and switching behaviour when it is operated close to the resonant frequency of the grating. This paper investigates the performance of such a grating for different pump beam intensities and different levels of gain and loss in the grating. The impact of gain/loss saturation on the grating response is also analysed. Operation of the grating as a logic gate, an amplifier and a switch is reported.

Throughout this paper, a time-domain numerical modelling method, namely the Transmission Line Modelling (TLM) method, is employed (Christopoulos 1995). The TLM method is based upon the analogy between the propagating electromagnetic field and voltage impulses travelling on an interconnected mesh of transmission lines. Successive repetitions of a scatter-propagate procedure, provide an explicit and stable time-stepping algorithm that mimics electromagnetic field behaviour to second order accuracy in both time and space (Christopoulos 1995; Hoefer 1985). It is important to note here, that the TLM method has been successfully implemented to model nonlinear and dispersive media (Paul et al. 2002; Paul et al. 1999). In our earlier work, we have validated the application of the TLM method to model PT Bragg gratings (Phang et al. 2013a) and also investigated the switching operation of the PT Bragg grating assuming a linear dielectric medium with a saturable non-dispersive gain and loss model (Phang et al. 2013b). However, any time-domain method, including the finite-difference time-domain (FDTD), could in principle be used.

This paper is organized as follows: the next section describes the PT grating structure considered in this paper together with the TLM model for dispersive and nonlinear dielectric materials, and saturable gain and loss. Section 3 reports on the 
performance of the structure for different levels of gain/loss and different intensities of the pump beam. Section 4 demonstrates possible applications of the structure as a logic gate, switch or amplifier driven by intensity.

\section{The Grating}

A schematic diagram of the PT Bragg grating considered in this paper is shown in Fig. 1(a) having a total length of $N \Lambda$, where $\Lambda$ is the grating period and $N$ is number of periods embedded in a medium with refractive index $n_{B}$. Fig. 1(b) shows one period of the grating with balanced gain and loss and 4 nonlinear layers having positive and negative Kerr nonlinearity. A grating structure with alternate layers of negative and positive Kerr nonlinearity, but without the inclusion of gain/loss, has been previously investigated by (Brzozowski and Sargent 2000) who proposed using inorganic or organic material; in this paper the magnitude of the Kerr nonlinearity assumed in our numeric investigations is typical of that of a GaAs based semiconductor material as reported by (Aitchison et al. 1997; Lan et al. 2004; Paré et al. 1996). The periodically modulated refractive index in such a grating can be expressed as,

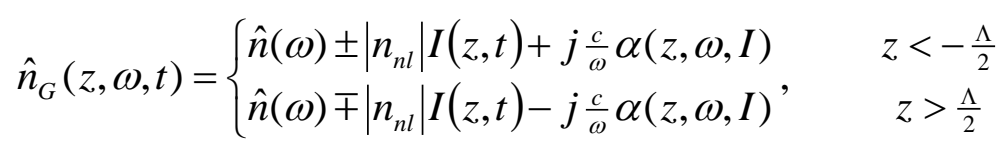

where $\hat{n}$ represents the base refractive index of the grating as a function of the frequency $\omega, n_{n l}$ is the Kerr nonlinearity constant, $I$ is the electric field intensity as a function of space and time and $\alpha$ denotes the gain/loss in the system. The refractive index profile of (1) has an intensity modulated real refractive index, i.e., for $I=0$, there is no modulation of the real part of the refractive index and the grating reduces to only alternating layers of gain and loss, Fig. 1(a). For $I \neq 0$ the refractive index of the grating is modulated by the intensity of the input signal, by effectively splitting each layer of gain and loss into two layers with positive and negative Kerr nonlinearity as shown in Fig. 1(b). We refer to such grating as Kerrnonlinearity induced PT Bragg grating (K-PTBG). The Bragg frequency $f_{B}$ is related to the average refractive index $\bar{n}$ of the structure by $f_{B}=\frac{c}{2 \bar{n} \Lambda}$ where $c$ is the speed of light in free-space. 


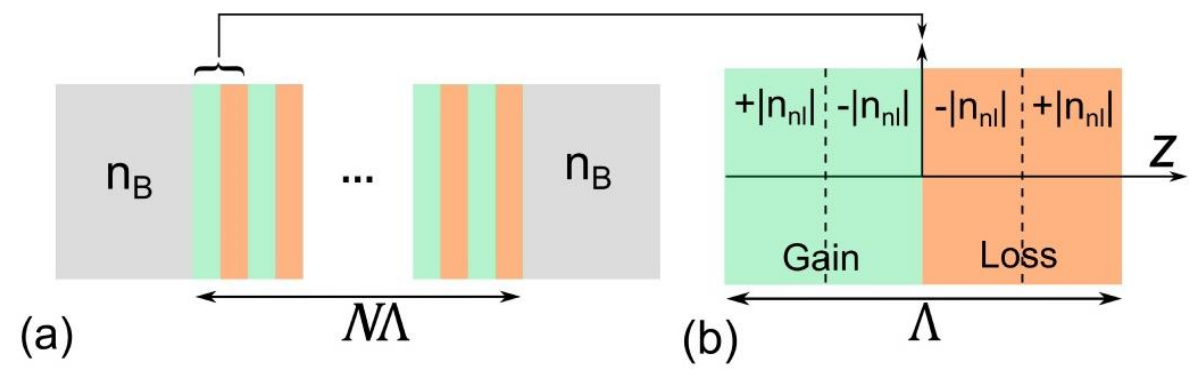

Fig. 1 Schematic of the PT grating (a) PT-grating with $I=0$, and (b) single period of the grating when $I \neq 0$.

Throughout the K-PTBG, a dispersive and intensity dependent medium defined by the Duffing model for field polarisation is implemented in the TLM method as in (Janyani et al. 2005; Janyani et al. 2004),

$$
\frac{\partial^{2} P_{D}}{\partial t^{2}}+2 \delta \frac{\partial P_{D}}{\partial t}+\omega_{0 D}^{2} P_{D} f_{D}=\varepsilon_{0} \chi_{e 0} \omega_{0 D}^{2} E
$$

where $P_{D}$ and $E$ are the Duffing polarization and electric field respectively, $\omega_{0 D}$ is the resonance frequency of the medium, $\delta$ is the damping constant, $\chi_{e 0}$ is the dielectric susceptibility at the zero frequency, $\varepsilon_{0}$ is the free-space permittivity and $f_{D}$ is the nonlinear Duffing function defined as (Janyani et al. 2005),

$$
f_{D}=e^{\varsigma\left|P_{D}\right|^{2}},
$$

with $\varsigma=-\frac{n_{n l} \sqrt{\chi_{c 0}+\chi_{e \infty}+1}}{\varepsilon_{0}^{2}\left(\chi_{e 0}+\chi_{e \infty}\right)^{3} \eta_{0}}$, where $n_{n l}$ is the Kerr-nonlinear constant, $\chi_{e \infty}$ is the electric susceptibility at the high-frequency limit and $\eta_{0}$ is the free-space impedance. It is important to note that in the absence of nonlinearity, i.e. $f_{D}=1$ the Duffing equation (2) reduces to the well-known linear dispersive classical model of dielectric material based on a simple harmonic oscillator with a Lorentzian profile, and therefore the refractive index of the medium at any frequency $\omega$ can be calculated as,

$$
\hat{n}(\omega)=\left(1+\chi_{e \infty}\right)+\frac{\chi_{e 0} \omega_{0 D}^{2}}{2 j \omega \delta+\left(\omega_{0 D}^{2}-\omega^{2}\right)} .
$$

The implementation and validation of the Duffing model for a dispersive and nonlinear optical medium in the TLM method has been reported in (Janyani et al. 2005; Janyani et al. 2004). 
In addition, a saturable and dispersive gain and loss model is also implemented in the TLM method as defined by (Hagness et al. 1996),

$$
|\alpha|(\omega, I)=\Omega(I)\left(\frac{\left|\alpha_{0}\right|}{1+j\left(\omega-\omega_{0 \sigma}\right) \tau}+\frac{\left|\alpha_{0}\right|}{1+j\left(\omega-\omega_{0 \sigma}\right) \tau}\right)
$$

where the gain/loss parameter $|\alpha|$ is related to the imaginary refractive index $n_{I}$ as $|\alpha|=\frac{\omega}{c}\left|n_{I}\right|, \quad \omega_{0 \sigma}$ denotes the atomic-transitional angular-frequency, $\tau$ is the dipole-relaxation time parameter, and $\alpha_{0}$ denotes the peak-value of gain or loss $\omega_{0 \sigma}$. The function $\Omega(I)$ denotes the saturation factor and is defined as,

$$
\Omega(I)=\frac{1}{1+\frac{I}{I_{S}}} .
$$

where $I$ and $I_{S}$ are the input and gain/loss saturation intensity, respectively. The saturation factor $\Omega$ is in the range $0<\Omega<1$, with $\Omega \rightarrow 1$, i.e. , $\frac{I}{I_{S}} \rightarrow 0$ referring to a non-saturated condition, and $\Omega \rightarrow 0$, i.e. $\frac{I}{I_{S}} \rightarrow \infty$, referring to a highly saturated condition. It is important to note that the model implemented, the Duffing equation and the gain and loss model, (2)-(6) both satisfy the KramersKronigs conditions which relate the real and imaginary parts of a complex refractive index.

\section{Results}

For definiteness the K-PTBG studied in this paper is based on GaAs material properties and comprises of 200 periods with the following material parameters, $\chi_{e \infty}=2.65, \chi_{e 0}=7.5, \omega_{0 D}=4614.4 \mathrm{rad} / \mathrm{ps}$, and $\delta=0.0923 \mathrm{rad} / \mathrm{ps}($ Bass et al. 2010). For the present numerical investigation the magnitude of the Kerr nonlinearity constant is also taken to be that of GaAs, $\left|n_{n l}\right|=2 \times 10^{-17} \mathrm{~m}^{2} \mathrm{~W}^{-1}$ (Aitchison et al. 1997; Lan et al. 2004; Paré et al. 1996) throughout the structure as shown in Fig. 1(b). However we note that in future experimental work other materials may be used to achieve alternating regions having Kerr coefficients of opposite sign (Brzozowski and Sargent 2000). The gain and loss parameters are $\tau=0.1 \mathrm{ps}$ and $\omega_{0 \sigma}=2116.5 \mathrm{rad} / \mathrm{ps}$ (Hagness et al. 1996), while the $\alpha_{0}$ depends on the gain or loss given. The periodicity of K-PTBG is designed so that the Bragg 
frequency is at the atomic-transitional frequency, i.e. $f_{B}=\frac{\omega_{0 \sigma}}{2 \pi}=336.85 \mathrm{THz}$, hence $\Lambda=122.7 \mathrm{~nm}$. The refractive index of the background material is again taken to be that of GaAs at $f_{B}$, i.e. $n_{B}=3.626$ (Bass et al. 2010).

The input beam comprises of two different beams, i.e. a probe beam and a strong pump beam. The pump beam is a continuous wave $(\mathrm{CW})$ and is used to alter the base refractive index of the grating through the Kerr nonlinearity. The frequency of the pump beam, $f_{\text {pump }}$, is set to be far from the Bragg frequency, i.e. $f_{\text {pump }}=200 \mathrm{THz}$, hence providing an uniform magnitude of real index modulation due to Kerr nonlinearity throughout the structure (Brzozowski and Sargent 2000). The probe beam is a Gaussian pulse modulated at the Bragg frequency $f_{B}$ and is low in intensity, with its maximum intensity being $1 \%$ of the pump beam intensity. Since the probe beam intensity is very low compared to the strong pump beam, its effect can be seen as a perturbation to the pump beam and hence the pump beam can be considered as the input beam.

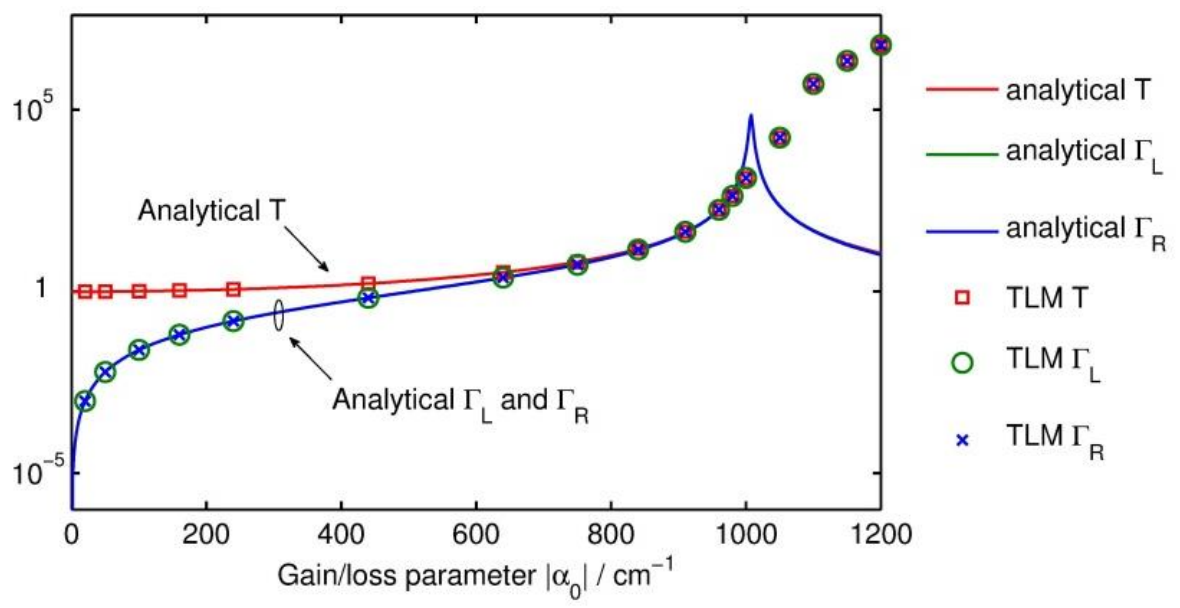

Fig. 2 Comparison of the transmittance and reflectance of a K-PTBG as a function of gain/loss parameter $\left|\alpha_{0}\right|$ obtained using the TLM and the analytical T-matrix method.

In order to identify the range of stable operation of the PTBG without the nonlinearity $\left(n_{n l}=0\right)$, the transmittance and reflectance of PTBG is analysed using both the analytical Transfer matrix (T-matrix) method (Collin 1991) and the TLM method. The T-matrix method is a frequency domain method that is limited to linear and dispersive structures, i.e. it cannot model Kerr nonlinearity and gain/loss saturation. The methodology of the T-matrix method is not presented in this paper and readers are referred to (Collin 1991). Fig. 2 shows the response of 
the PTBG grating assuming that the nonlinearity is switched off, i.e. $n_{n l}=0$, for different gain/loss parameters. Two sets of results are presented, namely the TLM results that include dispersive material model and saturation and the T-matrix results for a dispersive material model but no saturation. The saturation intensity is set to $I_{S}=2.5 \times 10^{13} \mathrm{Wm}^{-2}$ and the intensity of the pump and probe beams are set as described with the pump beam intensity of $I=2.5 \times 10^{11} \mathrm{Wm}^{-2}$. Fig. 2 shows the transmittance, $T$, and reflectance of a K-PTBG when excited from the left and right of the grating, $\Gamma_{L}$ and $\Gamma_{R}$ respectively, as a function of gain/loss parameter $\left|\alpha_{0}\right|$. The transmittance and reflectance denote transmitted and reflected power. The results are calculated at the Bragg frequency.

Fig. 2 shows that the reflectances of the K-PTBG with $n_{n l}=0$ are the same, $\Gamma_{L}=\Gamma_{R}$, regardless of the side of excitation. This is unlike the reported PTBG structures where the reflectances are different, and the results imply that this is due to the absence of the phase grating. The TLM results agree well with the Tmatrix results for low gain/loss parameter. As gain/loss in the structure increases, transmittance and reflectance also increase. The T-matrix results show a peak at $\left|\alpha_{0}\right| \approx 1008 \mathrm{~cm}^{-1}$ which is referred as the CPAL point for purely PT structures (no Kerr nonlinearity) and beyond which the lasing operation is observed (Nixon et al. 2012; Phang et al. 2013b). More importantly, as the only difference between the TLM and analytical results is the presence of the saturation in the TLM modes, it is observed that the TLM results do not exhibit a peak meaning that gain/loss saturation can limit the accumulation of energy inside the grating.

The response of the K-PTBG grating for different saturation intensities is analysed in Fig. 3(a,b) as a function of input intensity for three different cases namely, grating with no gain/loss,$\alpha_{0}=0$, with gain/loss parameter $\left|\alpha_{0}\right|=800 \mathrm{~cm}^{-1}$ and saturation turned off $(\Omega=1)$, and $\left|\alpha_{0}\right|=800 \mathrm{~cm}^{-1}$ with gain and loss saturation intensity $I_{S}=2.5 \times 10^{13} \mathrm{Wm}^{-2}$. In all cases Kerr nonlinearity is present with $\left|n_{n l}\right|=2 \times 10^{-17} \mathrm{~m}^{2} \mathrm{~W}^{-1}$. Fig. 3(a) shows the transmittance, $T$, which is the same regardless of the side of excitations. For the case $\alpha_{0}=0$, total transmittance $T=1$ is observed at low intensities but then transmittance gradually decreases to zero at high intensities. This can be explained by the fact that at high input 
intensities modulation of the refractive index due to Kerr nonlinearity becomes dominant and results in the formation of a Bragg grating. Since the structure for the case $\alpha_{0}=0$ is orthogonal (reciprocal and lossless), i.e. $T+\Gamma=1$, it follows that $\Gamma=0$ at low intensities and there is an almost-total reflectance $\Gamma \approx 1$ at high intensities (Fig. 3(b)).

For the case when the K-PTBG is operated with gain/loss parameter $\left|\alpha_{0}\right|=800 \mathrm{~cm}^{-1}$ and no saturation $(\Omega=1)$, the transmittance slowly increases and then decreases at higher input intensities. Fig. 3(b) shows that reflectance generally has different responses, $\Gamma_{L} \neq \Gamma_{R}$, depending on the side of excitation, with larger differences occurring at higher input intensities. At the intensity of $I_{U}=5.65 \times 10^{14} \mathrm{Wm}^{-2}$, unidirectional invisibility is observed, when $T=1$, reflectance from the right, $\Gamma_{R}$, is at its minimum, but $\Gamma_{L}$ is highly reflective (Phang et al. 2013b). This condition can be explained by the fact that at higher input intensities, the phase grating is induced by strong Kerr nonlinearity causing the reflectances to differ $\Gamma_{L} \neq \Gamma_{R}$ as observed in a linear PTBG (Phang et al. 2013b). It is noted that, when there is no saturation, the structure is dominated by PT symmetry at lower intensities. This shows that for a certain range of intensities $\left(I_{P T-K}<I<I_{2}\right)$ both PT and nonlinear behaviour are present and can be exploited simultaneously. At lower intensities PT behaviour is dominant, whereas nonlinear dynamics dominate at higher intensities. 


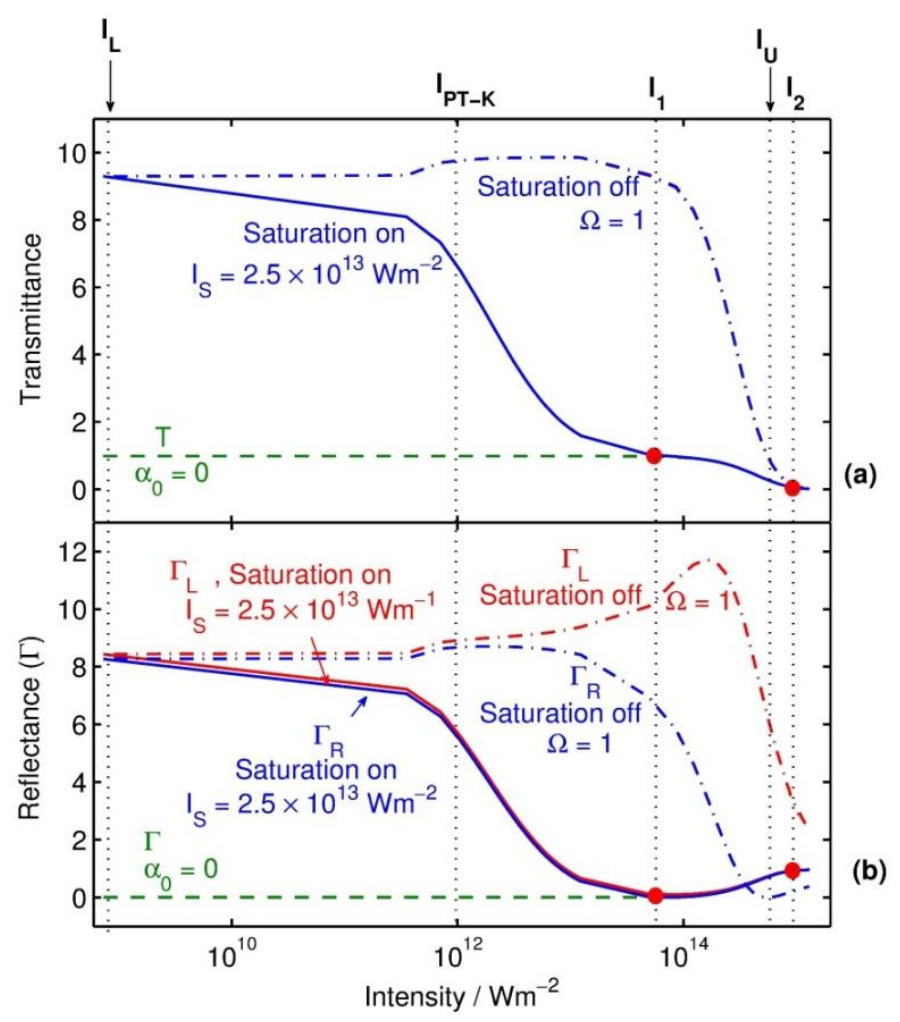

Fig. 3(a) Transmittance and (b) reflectances as a function of input beam intensity for no gain and loss $\left|\alpha_{0}\right|=0$; gain and loss $\left|\alpha_{0}\right|=800 \mathrm{~cm}^{-1}$ with no saturation $\Omega=1$; and gain and loss $\left|\alpha_{0}\right|=800 \mathrm{~cm}^{-1}$ with saturation intensity $I_{S}=2.5 \times 10^{13} \mathrm{Wm}^{-2}$.

In the case of $\left|\alpha_{0}\right|=800 \mathrm{~cm}^{-1}$ and $I_{S}=2.5 \times 10^{13} \mathrm{Wm}^{-2}$, the transmittance decreases as the intensity increases and after $I_{1}=5 \times 10^{13} \mathrm{Wm}^{-2}$ overlaps with the nonlinear Bragg grating with $\alpha_{0}=0$. Fig. 3(b) further shows that the reflectances for this case are the same regardless of the side of incidence. Similarly, the reflectances overlap with the one with no gain and loss $\left(\alpha_{0}=0\right)$ at very high intensities. This can be explained by the fact that saturation reduces the effective gain and loss in the structure, thus prohibiting PT behaviour at lower intensities. At higher intensities, above $I_{1}=5 \times 10^{13} \mathrm{Wm}^{-2}$, the behaviour of the K-PTBG corresponds to the nonlinear Bragg grating with no gain and loss. Therefore, low saturation intensity causes the gain and loss to saturate early and thus inhibits the non-reciprocal behaviour $\Gamma_{L} \neq \Gamma_{R}$ of a PTBG.

We now turn our attention to the region where we can manipulate both PT and nonlinear behaviour. Fig. 4 shows the frequency response, obtained by Fourier transformation of the time-domain signal, for the case in Fig. 3 when 
$\left|\alpha_{0}\right|=800 \mathrm{~cm}^{-1}$ and the gain and loss saturation is turned off $(\Omega=1)$ for three different input beam intensities, i.e. $I_{L}=7 \times 10^{8}, I_{U}=5.65 \times 10^{14}$, and $I_{2}=1 \times 10^{15}$ $\mathrm{Wm}^{-2}$, as marked in Fig. 3. The intensity of $I_{U}$ is when the unidirectional invisibility occurs as shown in Fig. 3. Results obtained by the T-matrix method are included for reference. Generally the transmittance is the same for the left and right incidence but reflectances differ for input intensities of $I_{U}$ and $I_{2}$. Fig. 4(a) shows the impact of input beam intensity on the spectra of the transmittance. The transmittance at low input intensity, $I_{L}$, fits with the one calculated using the Tmatrix method. However, at high input intensity,$I_{U}$, i.e., at the unidirectional invisibility operation, almost-total transmittance $T \approx 1$ is observed at the Bragg frequency. Further increase of the input intensity, increases the background index modulation even further which reduces the impact of the gain and loss resulting in a more defined band-gap. Theoretically, for a non-dispersive material with no gain/loss saturation the unidirectional operation is observed at all frequencies(Lin et al. 2011; Phang et al. 2013b). However, this result shows that the presence of the saturation and material dispersion reduces the U-operation to the vicinity of the Bragg frequency.

The reflectances $\Gamma_{L}$ and $\Gamma_{R}$ are shown in Fig. 4(b) and Fig. 4(c) respectively. For low input intensity , $I_{L}$, the TLM calculations agree with the results calculated by the T-matrix method, showing that the structure operates in a linear regime. However, when operated at the unidirectional point with intensity $I_{U}$, the reflectances are different, showing that the Kerr-nonlinearity induced a strong phase grating(Lin et al. 2011; Phang et al. 2013b). Further increase in input intensity to $I_{2}$ reduces the reflectance $\Gamma_{L}$ while increasing the reflectance $\Gamma_{R}$, which again can be explained by the fact that as the input intensity increases, the Kerr nonlinearity induced modulation of the background refractive index becomes more dominant and reduces the impact of gain and loss in the system resulting in a more pronounced band-gap property of the structure. 

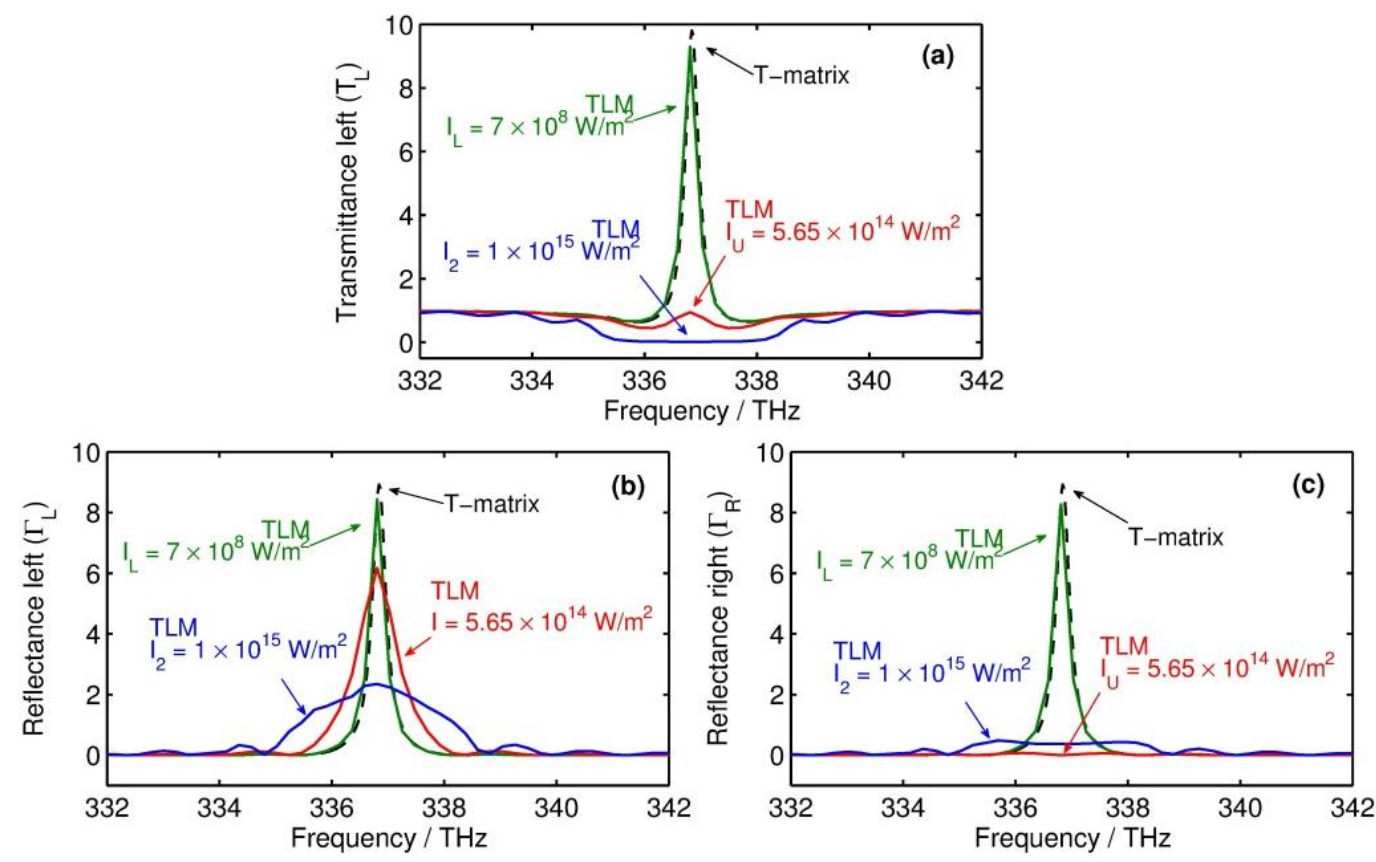

Fig. 4 (a) Transmittance $T$, (b) reflectance $\Gamma_{L}$ for signal incident from the left and (c) reflectance $\Gamma_{R}$ for signal incident from the right side of the grating, for three different input intensities namely, $I_{L}=7 \times 10^{8}, I_{U}=5.65 \times 10^{14}$, and $I_{2}=1 \times 10^{15} \mathrm{Wm}^{-2}$.

\section{Intensity driven all-optical signal processing device by Kerr-nonlinear PT Grating}

In this section, we investigate potential applications of the K-PTBG by controlling the level of the pump beam. The input signal comprises of both weak probe beam and strong pump beam. The probe beam is a CW signal operated at the Bragg frequency $f_{\text {probe }}=336.85 \mathrm{THz}$ with low intensity and is kept constant throughout the simulation with intensity $I_{\text {probe }}=1 \times 10^{6} \mathrm{Wm}^{-2}$. The pump beam is a CW signal operated far from the Bragg frequency at $f_{\text {pump }}=200 \mathrm{THz}$. The intensities of the pump beam are switched between two different values i.e., $I_{1}=5 \times 10^{13} \mathrm{Wm}^{-2}$ and $I_{2}=1 \times 10^{15} \mathrm{Wm}^{-2}$ as shown in Fig. 3(a). The gain/loss has a saturation intensity $I_{S}=2.5 \times 10^{13} \mathrm{Wm}^{-2}$ as in Fig. 3 .

Fig. 5(a) shows the pump beam as a function of time, initially turned off and then turned on to intensity $I_{1}$ for a duration of $10 \mathrm{ps}$, followed by increase in intensity to $I_{2}$ for another $10 \mathrm{ps,} \mathrm{and} \mathrm{then} \mathrm{repeating} \mathrm{the} \mathrm{same} \mathrm{pattern.} \mathrm{The} \mathrm{probe}$ beam is set to be constant throughout the simulation as shown in Fig. 5(b). Fig. 
5(c) shows the transmitted probe field when the grating is excited from the left. It can be seen that when the pump beam is switched off, the probe beam intensity is amplified by almost 10 times. Subsequent increase in the pump beam intensity to $I_{1}$ causes a decrease in the transmitted probe beam to the same value as the input probe beam $(T=1)$. When the pump beam intensity is increased to $I_{2}$, the transmitted probe field is significantly reduced.

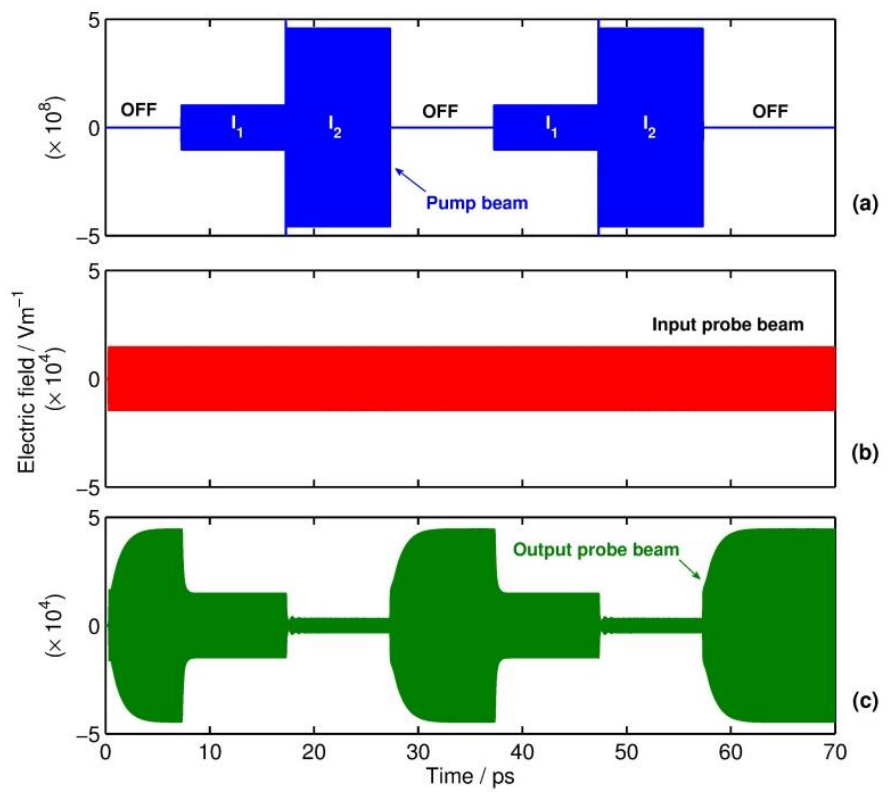

Fig. 5 Time domain response of the K-PTBG grating with the time domain response of (a) the pump beam and (b) the input probe beam and (c) the transmitted probe beam.

It is important to note that the change in response occurs almost instantaneously, with the longest switching time being 2.5 ps. Fig. 5(a,c) demonstrating that the K-PTBG can potentially be used as an optical amplifier (when the pump beam is turned off), optical switch (by using the pump beam to control the ON/OFF output of the probe beam) or as an optical negation logic gate, i.e. modulation of probe beam by the pump beam whereby the strong pump beam (logic 1) produces low probe beam (logic 0$)$.

\section{Conclusion}

This paper reports on a PT Bragg grating type of structure where the phase grating is formed by alternative layers of positive and negative Kerr nonlinearity. The analysis of the grating performance is based on the physical parameters of GaAs material that includes material dispersion and gain/loss saturation. The 
results show that a strong interplay of both PT and Kerr nonlinearity behaviour was only noted in the absence of the saturation. The presence of realistic GaAs gain/loss saturation can, at high input intensities, inhibit the PT characteristics of the grating. If both PT and nonlinear behaviour are to be preserved new materials with higher saturation levels need to be engineered. Nevertheless, we have demonstrated that GaAs K-PTBG with realistic gain/loss saturation can potentially be used for amplifier, optical switch or logic gate applications.

\section{References}

Achilleos, V., Kevrekidis, P.G., Frantzeskakis, D.J., Carretero-González, R.: Dark solitons and vortices in PT-symmetric nonlinear media: From spontaneous symmetry breaking to nonlinear PT phase transitions. Phys. Rev. A. 86, 013808 (2012).

Aitchison, J.S., Hutchings, D.C., Kang, J.U., Stegeman, G.I., Villeneuve, a.: The nonlinear optical properties of AlGaAs at the half band gap. IEEE J. Quantum Electron. 33, 341-348 (1997).

Bass, M., Li, G., van Stryland, E.: Handbook of optics vol. 4. McGraw Hill, New York, NY (2010).

Brzozowski, L., Sargent, E.H.: Optical signal processing using nonlinear distributed feedback structures. IEEE J. Quantum Electron. 36, 550-555 (2000).

Chong, Y.D., Ge, L., Stone, a. D.: PT-Symmetry Breaking and Laser-Absorber Modes in Optical Scattering Systems. Phys. Rev. Lett. 106, 093902 (2011).

Christopoulos, C.: The Transmission-Line Modeling Method TLM. IEEE Press, Piscataway (1995).

Collin, R.E.: Field theory of guided waves. IEEE Press, New York, NY (1991).

Feng, L., Wong, Z.J., Ma, R., Wang, Y., Zhang, X.: Parity-Time Synthetic Laser. arXiv Prepr. arXiv 1405.2863. 16 (2014).

Feng, L., Xu, Y.-L., Fegadolli, W.S., Lu, M.-H., Oliveira, J.E.B., Almeida, V.R., Chen, Y.-F., Scherer, A.: Experimental demonstration of a unidirectional reflectionless parity-time metamaterial at optical frequencies. Nat. Mater. 12, 108-13 (2013).

Hagness, S.C., Joseph, R.M., Taflove, A.: Subpicosecond electrodynamics of distributed Bragg reflector microlasers: Results from finite difference time domain simulations. Radio Sci. 31, 931-941 (1996).

Hodaei, H., Miri, M., Heinrich, M., Christodoulides, D.N., Khajavikhan, M.: PTsymmetric microring lasers: Self-adapting broadband mode-selective resonators. arXiv Prepr. arXiv 1405.2103. 12 (2014). 
Hoefer, W.J.R.: The Transmission-Line Matrix Method--Theory and Applications. IEEE Trans. Microw. Theory Tech. 33, 882-893 (1985).

Janyani, V., Vukovic, A., Paul, J.: The development of TLM models for nonlinear optics. Microw. Rev. 10, 35-42 (2004).

Janyani, V., Vukovic, A., Paul, J.D., Sewell, P., Benson, T.M.: Time domain simulation in photonics: A comparison of nonlinear dispersive polarisation models. Opt. Quantum Electron. 37, 3-24 (2005).

Kulishov, M., Laniel, J.M., Bélanger, N., Azaña, J., Plant, D. V: Nonreciprocal waveguide Bragg gratings. Opt. Express. 13, 3068-78 (2005).

Lan, S., Gopal, A.V., Kanamoto, K., Ishikawa, H.: Ultrafast response of photonic crystal atoms with Kerr nonlinearity to ultrashort optical pulses. Appl. Phys. Lett. 84, 5124 (2004).

Lin, Z., Ramezani, H., Eichelkraut, T., Kottos, T., Cao, H., Christodoulides, D.N.: Unidirectional Invisibility Induced by PT-Symmetric Periodic Structures. Phys. Rev. Lett. 106, 213901 (2011).

Makris, K.G., El-Ganainy, R., Christodoulides, D.N.: Beam Dynamics in PT Symmetric Optical Lattices. Phys. Rev. Lett. 100, 103904 (2008).

Musslimani, Z., Makris, K., El-Ganainy, R., Christodoulides, D.N.: Optical solitons in PT periodic potentials. Phys. Rev. Lett. 100, 030402 (2008).

Nixon, S., Ge, L., Yang, J.: Stability analysis for solitons in PT-symmetric optical lattices. Phys. Rev. A. 85, 023822 (2012).

Paré, C., Villeneuve, A., Bélanger, P.-A., Doran, N.J.: Compensating for dispersion and the nonlinear Kerr effect without phase conjugation. Opt. Lett. 21, 459 (1996).

Paul, J., Christopoulos, C., Thomas, D.W.P.: Generalized material models in TLM .I. Materials with frequency-dependent properties. IEEE Trans. Antennas Propag. 47, 1528-1534 (1999).

Paul, J., Christopoulos, C., Thomas, D.W.P.: Generalized material models in TLM - part 3: materials with nonlinear properties. IEEE Trans. Antennas Propag. 50, 997-1004 (2002).

Peng, B., Özdemir, Ş.K., Lei, F., Monifi, F., Gianfreda, M., Long, G.L., Fan, S., Nori, F., Bender, C.M., Yang, L.: Parity-time-symmetric whispering-gallery microcavities. Nat. Phys. 10, 1-33 (2014).

Phang, S., Vukovic, A., Susanto, H., Benson, T., Sewell, P.: Time Domain Modeling of All-Optical Switch based on PT-Symmetric Bragg Grating. 29th Annual Review of Progress in Applied Computational Electromagnetics. pp. 693698. Applied Computational Electromagnetics Society, Monterey, CA (2013)(a). 
Phang, S., Vukovic, A., Susanto, H., Benson, T.M., Sewell, P.: Ultrafast optical switching using parity-time symmetric Bragg gratings. J. Opt. Soc. Am. B. 30, 2984 (2013)(b).

Phang, S., Vukovic, A., Susanto, H., Benson, T.M., Sewell, P.: Impact of dispersive and saturable gain/loss on bistability of nonlinear parity-time Bragg gratings. Opt. Lett. 39, 2603-6 (2014).

Ramezani, H., Kottos, T., El-Ganainy, R., Christodoulides, D.N.: Unidirectional nonlinear PT-symmetric optical structures. Phys. Rev. A. 82, 043803 (2010).

Regensburger, A., Bersch, C., Miri, M.-A., Onishchukov, G., Christodoulides, D.N., Peschel, U.: Parity-time synthetic photonic lattices. Nature. 488, 167-71 (2012).

Rüter, C.E., Makris, K.G., El-Ganainy, R., Christodoulides, D.N., Segev, M., Kip, D.: Observation of parity-time symmetry in optics. Nat. Phys. 6, 192-195 (2010).

Schindler, J., Lin, Z., Lee, J.M., Ramezani, H., Ellis, F.M., Kottos, T.: \$lmathcal $\{$ PT $\}$ \$-symmetric electronics. J. Phys. A Math. Theor. 45, 444029 (2012).

Zhu, X., Feng, L., Zhang, P., Yin, X., Zhang, X.: One-way invisible cloak using parity-time symmetric transformation optics. Opt. Lett. 38, 2821-4 (2013). 